\title{
Grinding and Milling: Two Efficient Methodologies in the Solvent-Free Phosphomolybdic Acid-Catalyzed and Mechanochemical Synthesis of cis-4-Amido- $N$-yl-2-methyl-tetrahydroquinolines
}

\author{
Vladimir V. Kouznetsov, ${ }^{*, a}$ Diego R. Merchán-Arenas, ${ }^{b}$ Carlos A. Martínez-Bonilla, ${ }^{a}$ \\ Mario A. Macías, ${ }^{c}$ Pascal Roussel ${ }^{d}$ and Gilles H. Gauthier ${ }^{c}$ \\ ${ }^{a}$ Laboratorio de Química Orgánica y Biomolecular, Universidad Industrial de Santander, \\ Parque Tecnológico Guatiguara, Km 2 vía refugio, A.A. 681011 Piedecuesta, Colombia \\ ${ }^{b}$ Grupo de Investigación e Innovación en Básicas, Universidad Manuela Beltrán, Calle de los \\ Estudiantes 10-20 Ciudadela Real de Minas, A.A. 678 Bucaramanga, Colombia \\ ${ }^{c}$ Grupo INTERFASE, Universidad Industrial de Santander, Carrera 27, Calle 9, \\ Ciudad Universitaria, Bucaramanga, Colombia \\ ${ }^{d}$ Université Lille Nord de France, Unité de Catalyse et de Chimie du Solide, Equipe Chimie du \\ Solide, Avenue Dimitri Mendeleïev, Bâtiment C7, ENSCL/UST Lille 1, BP 90108, \\ 59652 Villeneuve d'Ascq Cedex, France
}

The ball-milling process of ABB' Povarov reaction through mechano-chemical activation with phosphomolybdic acid (PMA) as heteropolyacid (HPA) catalyst, was first time explored. The developed high speed vibratory ball milling (HSVBM) green method offers simple and efficient diastereoselective synthesis of cis-2,4-disubstituted tetrahydroquinolines in good to excellent yields $(56-76 \%)$.

Keywords: mechano-chemical milling, ABB' Povarov type reaction, phosphomolybdic acid catalyst, solvent-free Povarov reaction

\section{Introduction}

Several physical methods have been used to promote chemical reactions, including sonication, microwave, photocatalysis and the mechano-chemical approaches. The last one has become a valuable tool to promote many types of organic reactions in a ball-milling process, ${ }^{1,2}$ where the mechanical energy input is an important topic. At the same time this process depends on the mill type, ball material (quantity and size), scale of the synthesis, frequency, reaction time and energy dissipation. In this sense, the activation energy necessary to initiate a chemical reaction is directly related to the amount of energy in the system, and could be provided by grinding, shearing or extrusion. ${ }^{3}$ Of these three energy input ways, the grinding is the most useful technique which describes the mechanical action by hard surfaces and could be make it through manual or ball-milling method.

*e-mail: kouznet@uis.edu.co, vkuznechnik@gmail.com
In general, several types of reactions can be promoted by mechano-synthesis including metal mediated or catalyzed, condensation reactions, nucleophilic addition, cascade reactions, oxidations, halogenation, cycloaddition reaction, among others. ${ }^{1}$ Nonetheless, it should be noted that the mechano-chemical methodology has not been widely applied to promote the imino Diels-Alder (DA) reaction ${ }^{4}$ and remains as a great challenge in the organic synthesis field.

On the other hand, among several synthetic methodologies that aim reducing several waste and energy resources, ${ }^{5}$ domino and one pot processes have become the two of the most attractive ways to construct small bioactive heterocyclic molecules. For a long time, the DA reactions has been recognized as a valuable and powerful tool for producing bioactive six-membered carbo- and heterocycles through one step process. ${ }^{6-9}$ The semi-rigid tetrahydroquinolines (THQs) have been widely studied due to the biological relevance and considered the most notable of all the nitrogen containing heterocycles as well. 
Their rapid construction can be easily achieved via imino DA reaction (Povarov reaction), using a great variety of commercial anilines, aldehydes and electron-rich alkenes (dienophile). ${ }^{9-11}$ Recently, the scientific interest on the acid-catalyzed three component ABB' Povarov reaction has increased due to the possibility to generate, in a domino process, structurally differentiated and privileged 2,4-disubstituted THQs with pharmaceutical and new materials applications. ${ }^{12-14}$

However, there are still main drawbacks in the 2-MeTHQ synthesis via $\mathrm{ABB}$ ' reactions: expensive reagents including additives and catalysts, toxic and dangerous solvents, and energetic demand (reaction parameters reach until $\left.200{ }^{\circ} \mathrm{C}\right) \cdot{ }^{15,16} \mathrm{In}$ agreement with the current environmental concerns, to promote THQ system formation, designing and developing economically and environmentally benign synthesis, various physical methods as well as heterogeneous catalysis were recently proposed. ${ }^{17}$ Kavitha et al. ${ }^{18}$ reported the use of phosphotungstic acid (PWA, $\mathrm{H}_{3} \mathrm{PW}_{12} \mathrm{O}_{40}$ ) in the synthesis of $N$-(2-methyl-1,2,3,4tetrahydroquinolin-4-yl)pyrrolidin-2-ones via ABB' imino DA reaction employing $N$-vinyl pyrrolidin-4-one as a cyclic enamide. Therefore, in our research, we focused on the Diels-Alder catalyzed activity by other Brønsted acid potentially useful in our solvent free methodology, PMA, and in the scope of this study we didn't take into account the PWA activity, already used for this kind of reaction.

Looking for development of new methodologies to generate drug-like THQ-containing molecules under green reaction protocols, we took the possibility to use the mechano-chemical milling process on the imino DA reaction, this practical energy input way could be an important topic in our research program. ${ }^{19}$ Additionally, in spite of the 4-alkoxy(4-amido- $N$-yl)-2-methylTHQs synthesis has been carried out using a cascade methodology, ${ }^{20-23}$ the phosphomolybdic acid (PMA, $\mathrm{H}_{3} \mathrm{PMo}_{12} \mathrm{O}_{40}$ ) catalyzed using mechano-chemical milling to promote the Povarov reactions has not been investigated.

In this opportunity we want to report a new alternative and potential synthetic method of a straightforward way to build new series of 2-methyl-THQs using PMA-promoted mechano-chemical milling under solvent-free conditions.

\section{Experimental}

\section{Materials and methods}

All reagents were commercially obtained (synthesis degree) and were used without previous purification. A Fisher-Johns melting point apparatus was used to measure the melting points (uncorrected). Lumex Infralum FT-02 spectrophotometer was used to measure the IR spectra in $\mathrm{KBr}$ discs. NMR (1D and 2D) spectra were recorded on Bruker Avance-400 spectrometer.

For crystal structure determination, the data were collected on a Bruker APEX2 diffractometer using a graphitemonochromatized Mo $\mathrm{K} \alpha$ radiation $(\lambda=0.71073 \AA)$. An Agilent Technologies 6890 plus Gas Chromatograph interfaced to an Agilent Technologies MSD 5963 Selective Detector (MSD) was used for MS identification at $70 \mathrm{eV}$ using a $60 \mathrm{~m}$ capillary column coated with HP-5 [5\%-phenyl-poly(dimethyl-siloxane)]. Elemental analyses were performed on a PerkinElmer 2400 Series II analyzer and were within \pm 0.4 of theoretical values.

General procedure for the synthesis of $3 a$ under high speed vibratory ball mill (HSVBM)

The aniline (1a) was added ( $1 \mathrm{mmol}, 0.24 \mathrm{~g})$ with $N$-vinyl amide (2a, $N$-vinyl acetamide, $2.1 \mathrm{mmol}, 0.25 \mathrm{~g}$ ) and PMA (10 mol\%, $0.30 \mathrm{~g})$ into the open air agate dish and one ball $(\mathrm{d}=51.6 \mathrm{~mm}, 186.25 \mathrm{~g})$ was put over the mixture, the mill was closed and the reaction was launched to the maximum mill frequency $(60 \mathrm{~Hz})$. After full conversion (TLC), around $3 \mathrm{~h}$, the mixture was easily removed and dissolved in ethyl acetate $(20 \mathrm{~mL})$. Afterward, the solution was quenched with a saturated solution of $\mathrm{NaHCO}_{3}$, extracted with ethyl acetate, dried over anhydrous $\mathrm{Na}_{2} \mathrm{SO}_{4}$ and concentrated under reduced pressure. The crude product was purified by column chromatography on silica gel using a mixture of petroleum ether and ethyl acetate as eluent to afford the THQ derivative $\mathbf{3 a}$.

\section{X-Ray crystallography}

The data were collected on a Bruker APEX2 diffractometer using a graphite-monochromatized Mo $K \alpha$ radiation $(\lambda=0.71073 \AA)$. After data collection, the intensity data were integrated and corrected for Lorentz polarization and background effects using the SAINT V7.34A software. The SADABS-2012/1 software was used to correct absorption effects using a redundancy algorithm. Crystal structure was determined by direct methods using the SIR92 program and refined using SHELXL2014. Crystal data, data collection and structure refinement details are summarized in Table S1 (Supplementary Information). Anisotropic displacement parameters were refined for C, $\mathrm{N}$ and $\mathrm{O}$ atoms. All $\mathrm{H}$ atoms (constrained to ride on their parent atoms) were placed in geometrically idealized positions. The used $\mathrm{C}-\mathrm{H}$ mean distances were $0.93 \AA$ (aromatic) and 0.96-0.97 $\AA$ (methyl), while a $0.856 \AA$ value was used for the N-H distances. 
General procedure for the synthesis of $T H Q$ derivatives under high speed mixer ball mill (HSMBM)

Reactions were carried out in Wig-L-Bug mixer mill at a frequency of $80 \mathrm{~Hz}$ using a $2.5 \mathrm{~mL}$ stainless steel grinding jar and a single ball $(\mathrm{d}=10 \mathrm{~mm}, 1.05 \mathrm{~g})$ of the same material. The suitable aryl amine ( $1 \mathrm{mmol}), N$-vinyl amide $(2.1 \mathrm{mmol})$ and PMA (10 mol\%) were added to a ball mill vessel with a single stainless steel ball. The vessel was fixed to a horizontal mixer arm and it was allowed to mix for $1 \mathrm{~h}$ at a frequency of $80 \mathrm{~Hz}$ (longer times generate the substrate decomposition). Then, the stuck reaction mixture was washed with ethanol/ethyl acetate (1:3) mixture and concentrated under reduced pressure. The reaction mixture was quenched with a saturated solution of $\mathrm{NaHCO}_{3}$, extracted with ethyl acetate, dried over anhydrous $\mathrm{Na}_{2} \mathrm{SO}_{4}$ and concentrated under reduced pressure. The evaporation gave the crude product, which was purified by column chromatography on silica gel using a mixture of petroleum ether and ethyl acetate as eluent to afford the corresponding THQ derivatives 3a-i (see Supplementary Information).

cis- $N$-(6-Acetyl-2-methyl-1,2,3,4-tetrahydroquinolin-4-yl) acetamide (3a)

White solid; $65 \%$ yield; mp $225-228{ }^{\circ} \mathrm{C}$; IR (KBr) $v / \mathrm{cm}^{-1} 3363,3239,3070,1635,1589,1434,1280$, 817; ${ }^{1} \mathrm{H}$ NMR (400 MHz, DMSO- $d_{6}$ ) $\delta 1.17$ (d, 3H, $\left.J 6.3 \mathrm{~Hz}, 2-\mathrm{CH}_{3}\right), 1.38\left(\mathrm{q}, 1 \mathrm{H}, J 11.5 \mathrm{~Hz}, 3-\mathrm{H}_{\mathrm{ax}}\right), 1.94$ (s, $\left.3 \mathrm{H}, 4-\mathrm{COCH}_{3}\right), 2.02-1.96\left(\mathrm{~m}, 1 \mathrm{H}, 3-\mathrm{H}_{\mathrm{eq}}\right), 2.35(\mathrm{~s}, 3 \mathrm{H}$, 6- $\left.\mathrm{COCH}_{3}\right), 3.55\left(\mathrm{dqd}, 1 \mathrm{H}, J 11.5,6.3,2.6 \mathrm{~Hz}, 2-\mathrm{H}_{\mathrm{ax}}\right)$, 5.02 (ddd, 1H, J 11.5, 8.8, $5.4 \mathrm{~Hz}, 4-\mathrm{Hax}), 6.48$ (d, 1H, $\left.J 8.3 \mathrm{~Hz}, 8-\mathrm{H}_{\mathrm{Ar}}\right), 6.73(\mathrm{~s}, 1 \mathrm{H}, 1-\mathrm{NH}), 7.52\left(\mathrm{~s}, 1 \mathrm{H}, 5-\mathrm{H}_{\mathrm{Ar}}\right)$, 7.55 (d, 1H, J 8.3 Hz, 7-HAr), 8.18 (d, 1H, J 8.8 Hz, 4-NH); ${ }^{13} \mathrm{C}$ NMR (100 MHz, DMSO- $\left.d_{6}\right) \delta 21.5,22.8,25.8,36.6$, 44.9, 45.9, 112.0, 120.2, 124.4, 127.2, 129.3, 149.9, 169.4, 194.9; GC-MS (70 eV), $t_{\mathrm{R}} 25.1 \mathrm{~min}, \mathrm{~m} / \mathrm{z}$ (relative intensity) $[\mathrm{M}]^{+} 246$ (10), 231 (5), 215 (2), 203 (12), 186 (80), 172 (100). Anal. calcd. for $\mathrm{C}_{14} \mathrm{H}_{18} \mathrm{~N}_{2} \mathrm{O}_{2}: \mathrm{C}, 68.27 ; \mathrm{H}, 7.37 ; \mathrm{N}$, 11.37. Found: C, 68.55; H, 7.12; N, 11.20.

cis- $N$-(2-Methyl-1,2,3,4-tetrahydroquinolin-4-yl)acetamide (3b)

White solid; $56 \%$ yield; mp $127-130{ }^{\circ} \mathrm{C}$; IR (KBr) $v / \mathrm{cm}^{-1} 3347,3255,3070,2962,1635,1496,1311,971$, 755; ${ }^{1} \mathrm{H}$ NMR (400 MHz, $\left.\mathrm{CDCl}_{3}\right) \delta 1.12(\mathrm{~d}, 3 \mathrm{H}, J 6.2 \mathrm{~Hz}$, $\left.2-\mathrm{CH}_{3}\right), 1.38\left(\mathrm{q}, 1 \mathrm{H}, J 11.8 \mathrm{~Hz}, 3-\mathrm{H}_{\mathrm{ax}}\right), 1.88(\mathrm{~s}, 3 \mathrm{H}$, 4- $\left.\mathrm{COCH}_{3}\right), 1.95-1.89\left(\mathrm{~m}, 1 \mathrm{H}, 3-\mathrm{H}_{\mathrm{eq}}\right), 3.44-3.38(\mathrm{~m}, 1 \mathrm{H}$, $2-\mathrm{H}_{\mathrm{ax}}$ ), 5.02 (ddd, $\left.1 \mathrm{H}, J 11.8,8.9,6.0 \mathrm{~Hz}, 4-\mathrm{H}_{\mathrm{ax}}\right), 5.66$ (s, $1 \mathrm{H}, 1-\mathrm{NH}), 6.49-6.44\left(\mathrm{~m}, 2 \mathrm{H}, 7-\mathrm{H}_{\mathrm{Ar}}, 5-\mathrm{H}_{\mathrm{Ar}}\right), 6.92-6.86(\mathrm{~m}$, $\left.2 \mathrm{H}, 6-\mathrm{H}_{\mathrm{Ar}}, 8-\mathrm{H}_{\mathrm{Ar}}\right), 8.12(\mathrm{~d}, 1 \mathrm{H}, J 8.9 \mathrm{~Hz}, 4-\mathrm{NH}) ;{ }^{13} \mathrm{C} \mathrm{NMR}$ $\left(100 \mathrm{MHz}, \mathrm{CDCl}_{3}\right) \delta 21.9,22.7,37.5,45.1,46.0,113.4$,
115.5, 121.3, 126.6, 127.4, 145.7, 169.2; GC-MS (70 eV), $t_{\mathrm{R}} 16.7 \mathrm{~min}, \mathrm{~m} / z$ (relative intensity) $[\mathrm{M}]^{+} 204$ (25), 187 (2), 173 (2), 161 (5), 114 (60), 130 (100), 77 (5). Anal. calcd. for $\mathrm{C}_{12} \mathrm{H}_{16} \mathrm{~N}_{2} \mathrm{O}$ : C, 70.56; H, 7.90; N, 13.71. Found: C, $70.71 ; \mathrm{H}, 7.69 ; \mathrm{N}, 13.55$.

cis- $N$-(6-Methoxy-2-methyl-1,2,3,4-tetrahydroquinolin-4-yl) acetamide (3c)

White solid; $64 \%$ yield; mp $155-158{ }^{\circ} \mathrm{C}$; IR (KBr) $\mathrm{v} / \mathrm{cm}^{-1} 3378,3286,2962,1727,1635,1542,1295,1234$, $1157,1033,817 ;{ }^{1} \mathrm{H}$ NMR (400 MHz, DMSO- $\left.d_{6}\right) \delta 1.10$ (d, $\left.3 \mathrm{H}, J 6.0 \mathrm{~Hz}, 2-\mathrm{CH}_{3}\right), 1.36\left(\mathrm{q}, 1 \mathrm{H}, J 11.7 \mathrm{~Hz}, 3-\mathrm{H}_{\mathrm{ax}}\right), 1.89$ (s, $\left.3 \mathrm{H}, 6-\mathrm{COCH}_{3}\right), 1.96-1.90\left(\mathrm{~m}, 1 \mathrm{H}, 3-\mathrm{H}_{\mathrm{eq}}\right), 3.30-3.37(\mathrm{~m}, 1 \mathrm{H}$, $\left.2-\mathrm{H}_{\mathrm{ax}}\right), 3.60\left(\mathrm{~s}, 3 \mathrm{H}, 6-\mathrm{OCH}_{3}\right), 5.05-4.98\left(\mathrm{~m}, 1 \mathrm{H}, 4-\mathrm{H}_{\mathrm{ax}}\right), 5.28$ (s, 1H, 1-NH), $6.43\left(\mathrm{~d}, 1 \mathrm{H}, J 8.5 \mathrm{~Hz}, 8-\mathrm{H}_{\mathrm{Ar}}\right), 6.51(\mathrm{~s}, 1 \mathrm{H}$, $\left.5-\mathrm{H}_{\mathrm{Ar}}\right), 6.58\left(\mathrm{~d}, 1 \mathrm{H}, J 8.5 \mathrm{~Hz}, 7-\mathrm{H}_{\mathrm{Ar}}\right), 8.15(\mathrm{~d}, 1 \mathrm{H}, J 8.7 \mathrm{~Hz}$, $4-\mathrm{NH}) ;{ }^{13} \mathrm{C}$ NMR $\left(100 \mathrm{MHz}, \mathrm{DMSO}-d_{6}\right) \delta 22.0,22.7,37.7$, 45.4, 46.1, 55.3, 112.4, 113.6, 114.6, 122.6, 140.1, 150.6, 169.2; GC-MS (70 eV), $t_{\mathrm{R}} 20.7 \mathrm{~min}, \mathrm{~m} / z$ (relative intensity) $[\mathrm{M}]^{+} 234$ (25), 234 (25), 219 (2), 203 (2), 174 (55), 160 (100). Anal. calcd. for $\mathrm{C}_{13} \mathrm{H}_{18} \mathrm{~N}_{2} \mathrm{O}_{2}: \mathrm{C}, 66.64 ; \mathrm{H}, 7.74 ; \mathrm{N}$, 11.96. Found: C, 66.53; H, 7.92; N, 11.75.

cis- $N$-(6-Acetyl-2-methyl-1,2,3,4-tetrahydroquinolin-4-yl) formamide (3d)

Pale yellow solid; $76 \%$ yield; $\mathrm{mp} 235-237^{\circ} \mathrm{C}$; IR $(\mathrm{KBr})$ v / $\mathrm{cm}^{-1}$ 3348, 3255, 3039, 1650, 1589, 1434, 1249, 817; ${ }^{1} \mathrm{H}$ NMR (400 MHz, DMSO- $\left.d_{6}\right) \delta 1.18(\mathrm{~d}, 3 \mathrm{H}, J 6.3 \mathrm{~Hz}$, $\left.2-\mathrm{CH}_{3}\right), 1.42\left(\mathrm{q}, 1 \mathrm{H}, J 12.2 \mathrm{~Hz}, 3-\mathrm{H}_{\mathrm{ax}}\right), 2.04$ (td, $1 \mathrm{H}, J$ 12.2, 4.2, $\left.2.1 \mathrm{~Hz}, 3-\mathrm{H}_{\mathrm{eq}}\right), 2.35\left(\mathrm{~s}, 3 \mathrm{H}, 6-\mathrm{COCH}_{3}\right), 3.55$ (dqd, $\left.1 \mathrm{H}, J 12.2,6.3,2.1 \mathrm{~Hz}, 2-\mathrm{H}_{\mathrm{ax}}\right), 5.12(\mathrm{ddd}, 1 \mathrm{H}, J 12.2,9.3$, $\left.4.2 \mathrm{~Hz}, 4-\mathrm{H}_{\mathrm{ax}}\right), 6.51-6.42\left(\mathrm{~m}, 2 \mathrm{H}, 1-\mathrm{NH}, 8-\mathrm{H}_{\mathrm{Ar}}\right), 7.51(\mathrm{dd}$, $\left.1 \mathrm{H}, J 8.5,1.3 \mathrm{~Hz}, 7-\mathrm{H}_{\mathrm{Ar}}\right), 7.56\left(\mathrm{~d}, 1 \mathrm{H}, J 1.3 \mathrm{~Hz}, 5-\mathrm{H}_{\mathrm{Ar}}\right), 8.23$ (s, $1 \mathrm{H}, 4-\mathrm{COH}), 8.28$ (d, $1 \mathrm{H}, J 9.3 \mathrm{~Hz}, 4-\mathrm{NH}) ;{ }^{13} \mathrm{C} \mathrm{NMR}$ $\left(100 \mathrm{MHz}, \mathrm{DMSO}-d_{6}\right) \delta 21.2,25.5,36.3,43.5,45.7,112.0$, 119.0, 124.3, 127.1, 128.9, 149.6, 160.9, 194.5; CG-MS $(70 \mathrm{eV}), t_{\mathrm{R}} 24.3 \mathrm{~min}, \mathrm{~m} / \mathrm{z}$ (relative intensity) $[\mathrm{M}]^{+} 232(10)$, 217 (10), 186 (60), 172 (100), 161 (10), 144 (25), 129 (8). Anal. calcd. for $\mathrm{C}_{13} \mathrm{H}_{16} \mathrm{~N}_{2} \mathrm{O}_{2}$ : C, 67.22; H, 6.94; N, 12.06 . Found: C, 67.45; H, 6.69; N, 12.34.

cis-N-(2-Methyl-1,2,3,4-tetrahydroquinolin-4-yl)formamide (3e)

White solid; $63 \%$ yield; mp $158-160{ }^{\circ} \mathrm{C}$; IR (KBr) $v / \mathrm{cm}^{-1} 3286,3023,1666,1527,1496,755 ;{ }^{1} \mathrm{H}$ NMR

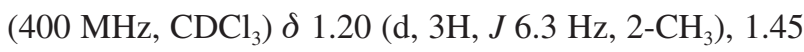
$\left(\mathrm{q}, 1 \mathrm{H}, J 11.4 \mathrm{~Hz}, 3-\mathrm{H}_{\mathrm{ax}}\right), 2.26(\mathrm{ddd}, 1 \mathrm{H}, J 11.4,6.2,2.1 \mathrm{~Hz}$, $\left.3-\mathrm{H}_{\mathrm{eq}}\right), 3.53\left(\mathrm{dqd}, 1 \mathrm{H}, J 11.4,6.3,2.1 \mathrm{~Hz}, 2-\mathrm{H}_{\mathrm{ax}}\right), 3.78$ (s, $1 \mathrm{H}, 1-\mathrm{NH}), 5.39$ (ddd, $1 \mathrm{H}, J 11.4,8.4,6.2 \mathrm{~Hz}, 4-\mathrm{H}_{\mathrm{ax}}$ ), 5.87 (d, $1 \mathrm{H}, J 8.4 \mathrm{~Hz}, 4-\mathrm{NH}), 6.49$ (dd, $\left.1 \mathrm{H}, J 7.5,0.6 \mathrm{~Hz}, 8-\mathrm{H}_{\mathrm{Ar}}\right)$, $6.67\left(\mathrm{td}, 1 \mathrm{H}, J 7.5,0.8 \mathrm{~Hz}, 7-\mathrm{H}_{\mathrm{Ar}}\right), 7.03(\mathrm{td}, 1 \mathrm{H}, J 7.7,7.5$, 
$\left.0.6 \mathrm{~Hz}, 6-\mathrm{H}_{\mathrm{Ar}}\right), 7.10\left(\mathrm{dd}, 1 \mathrm{H}, J\right.$ 7.7, $\left.0.8 \mathrm{~Hz}, 5-\mathrm{H}_{\mathrm{Ar}}\right), 8.29$ (s, $1 \mathrm{H}, 4-\mathrm{COH}) ;{ }^{13} \mathrm{C}$ NMR $\left(100 \mathrm{MHz}, \mathrm{CDCl}_{3}\right) \delta 22.2$, 37.9, 44.9, 46.6, 114.5, 117.8, 120.8, 127.0, 128.5, 145.3, 161.3; GC-MS (70 eV), $t_{\mathrm{R}} 16.7 \mathrm{~min}, \mathrm{~m} / z$ (relative intensity) $[\mathrm{M}]^{+} 190$ (25), 161 (10), 144 (45), 130 (100), 77 (15). Anal. calcd. for $\mathrm{C}_{11} \mathrm{H}_{14} \mathrm{~N}_{2} \mathrm{O}: \mathrm{C}, 69.45 ; \mathrm{H}, 7.42 ; \mathrm{N}, 14.73$. Found: C, 69.21; H, 7.55; N, 14.59.

cis-N-(6-Methoxy-2-methyl-1,2,3,4-tetrahydroquinolin-4-yl) formamide (3f)

White solid; $60 \%$ yield; mp $151-153{ }^{\circ} \mathrm{C}$; IR (KBr) $\mathrm{v} / \mathrm{cm}^{-1}$ 3286, 3239, 3023, 2838, 1666, 1542, 1265, 1234, 1033, 725; ${ }^{1} \mathrm{H}$ NMR (400 MHz, DMSO- $\left.d_{6}\right) \delta 1.11$ (d, $3 \mathrm{H}$, $\left.J 5.7 \mathrm{~Hz}, 2-\mathrm{CH}_{3}\right), 1.39$ (q, $1 \mathrm{H}, J 11.5 \mathrm{~Hz}, 3-\mathrm{H}_{\mathrm{ax}}$ ), $1.98-$ $1.94\left(\mathrm{~m}, 1 \mathrm{H}, 3-\mathrm{H}_{\mathrm{eq}}\right), 3.36-3.34\left(\mathrm{~m}, 1 \mathrm{H}, 2-\mathrm{H}_{\mathrm{ax}}\right), 3.60(\mathrm{~s}$, $3 \mathrm{H}, 6-\mathrm{OCH}_{3}$ ), 5.12 (ddd, $1 \mathrm{H}, J 11.5,8.4,3.5 \mathrm{~Hz}, 4-\mathrm{H}_{\mathrm{ax}}$ ), $5.30(\mathrm{~s}, 1 \mathrm{H}, 1-\mathrm{NH}), 6.45\left(\mathrm{~d}, 1 \mathrm{H}, J 8.4 \mathrm{~Hz}, 8-\mathrm{H}_{\mathrm{Ar}}\right), 6.53$ $\left(\mathrm{s}, 1 \mathrm{H}, 5-\mathrm{H}_{\mathrm{Ar}}\right), 6.59\left(\mathrm{~d}, 1 \mathrm{H}, J 8.4 \mathrm{~Hz}, 7-\mathrm{H}_{\mathrm{Ar}}\right), 8.19(\mathrm{~s}, 1 \mathrm{H}$, 4-COH), 8.35 (d, 1H, J 8.4 Hz, 4-NH); ${ }^{13} \mathrm{C} \mathrm{NMR} \mathrm{(100} \mathrm{MHz,}$ DMSO- $\left.d_{6}\right) \delta 21.9,37.5,44.2,46.3,55.3,112.1,113.9$, $114.8,121.8,140.1,150.7,161.1$; CG-MS (70 eV), $t_{\mathrm{R}} 20.4$ $\min , m / z$ (relative intensity) $[\mathrm{M}]^{+} 220$ (35), 160 (100), 145 (10), 130 (15), 117 (15). Anal. calcd. for $\mathrm{C}_{12} \mathrm{H}_{16} \mathrm{~N}_{2} \mathrm{O}_{2}$ : C, 65.43; H, 7.32; N, 12.72. Found: C, 65.61; H, 7.11; N, 12.76 .

cis-1-(6-Acetyl-2-methyl-1,2,3,4-tetrahydroquinolin-4-yl) pyrrolidin-2-one $\mathbf{( 3 g})$

White solid; $69 \%$ yield; mp $165-167{ }^{\circ} \mathrm{C}$; IR (KBr) $\mathrm{v} / \mathrm{cm}^{-1}$ 3332, 2962, 2915, 2885, 1681, 1573, 1434, 956, 833; ${ }^{1} \mathrm{H}$ NMR (400 MHz, DMSO- $\left.d_{6}\right) \delta 1.19(\mathrm{~d}, 3 \mathrm{H}$, $\left.J 6.3 \mathrm{~Hz}, 2-\mathrm{CH}_{3}\right), 1.63\left(\mathrm{q}, 1 \mathrm{H}, J 11.9 \mathrm{~Hz}, 3-\mathrm{H}_{\mathrm{ax}}\right), 1.81$ (ddd, $1 \mathrm{H}, J$ 11.9, 5.2, $2.3 \mathrm{~Hz}, 3-\mathrm{H}_{\mathrm{eq}}$ ), 2.02-1.89 (m, 2H, 4'- $\mathrm{H}_{\mathrm{a}}$, $\left.4^{\prime}-\mathrm{H}_{\mathrm{b}}\right), 2.36$ (s, 3H, 6- $\left.\mathrm{COCH}_{3}\right), 2.41-2.34\left(\mathrm{~m}, 2 \mathrm{H}, 3\right.$ ' $-\mathrm{H}_{\mathrm{a}}$, $\left.3^{\prime}-\mathrm{H}_{\mathrm{b}}\right), 3.03-2.96\left(\mathrm{~m}, 1 \mathrm{H}, 5^{\prime}-\mathrm{H}_{\mathrm{a}}\right), 3.26-3.19\left(\mathrm{~m}, 1 \mathrm{H}, 5^{\prime}-\mathrm{H}_{\mathrm{b}}\right)$, 3.61 (ddd, 1H, J 11.9, 6.3, $\left.2.3 \mathrm{~Hz}, 2-\mathrm{H}_{\mathrm{ax}}\right), 5.25(\mathrm{dd}, 1 \mathrm{H}$, $\left.J 11.9,5.2 \mathrm{~Hz}, 4-\mathrm{H}_{\mathrm{ax}}\right), 6.53\left(\mathrm{~d}, 1 \mathrm{H}, J 8.5 \mathrm{~Hz}, 8-\mathrm{H}_{\mathrm{Ar}}\right), 6.77$ (s, 1H, 1-NH), $7.21\left(\mathrm{~s}, 1 \mathrm{H}, 5-\mathrm{H}_{\mathrm{Ar}}\right), 7.57(\mathrm{dd}, 1 \mathrm{H}, J$ 8.5, $\left.1.7 \mathrm{~Hz}, 7-\mathrm{H}_{\mathrm{Ar}}\right) ;{ }^{13} \mathrm{C} \mathrm{NMR}\left(100 \mathrm{MHz}, \mathrm{DMSO}-d_{6}\right) \delta 18.0$, 21.4, 25.9, 30.8, 33.0, 41.6, 45.9, 47.1, 112.5, 116.7, 124.6, $126.3,129.4,150.5,174.8,194.9$; GC-MS (70 eV), $t_{\mathrm{R}} 23.6$ $\mathrm{min}, \mathrm{m} / z$ (relative intensity) $[\mathrm{M}]^{+} 272(5), 257$ (3), 243(3), 187 (65), 172 (100). Anal. calcd. for $\mathrm{C}_{16} \mathrm{H}_{20} \mathrm{~N}_{2} \mathrm{O}_{2}$ : C, 70.56; H, 7.40; N, 12.16. Found: C, 70.72; H, 7.27; N, 12.25.

cis-1-(2-Methyl-1,2,3,4-tetrahydroquinolin-4-yl)pyrrolidin2-one (3h)

White solid; $67 \%$ yield; mp 95-97 ${ }^{\circ} \mathrm{C}$; IR (KBr) $\mathrm{v} / \mathrm{cm}^{-1}$ 3317, 3039, 2977, 2946, 2885, 1666, 1604, 1496, 1265,$755 ;{ }^{1} \mathrm{H}$ NMR (400 MHz, DMSO- $\left.d_{6}\right) \delta 1.15$ (d, 3H, $\left.J 6.2 \mathrm{~Hz}, 2-\mathrm{CH}_{3}\right), 1.62$ (q, $1 \mathrm{H}, J 11.8 \mathrm{~Hz}, 3-\mathrm{H}_{\mathrm{ax}}$ ), 1.75 (ddd, $\left.1 \mathrm{H}, J 11.8,5.7,2.1 \mathrm{~Hz}, 3-\mathrm{H}_{\mathrm{eq}}\right), 1.96-1.87\left(\mathrm{~m}, 2 \mathrm{H}, 4^{\prime}-\mathrm{H}_{\mathrm{a}}\right.$, $\left.4^{\prime}-\mathrm{H}_{\mathrm{b}}\right), 2.42-2.26\left(\mathrm{~m}, 2 \mathrm{H}, 3^{\prime}-\mathrm{H}_{\mathrm{a}}, 3^{\prime}-\mathrm{H}_{\mathrm{b}}\right), 2.95$ (dt, 1H, J9.4, $6.7 \mathrm{~Hz}, 5$ '- $\mathrm{H}_{\mathrm{a}}$ ), 3.19 (dt, 1H, J9.4, 7.2 Hz, 5'-- ${ }_{\mathrm{b}}$ ), 3.48 (dqd, $\left.1 \mathrm{H}, J 11.8,6.2,2.1 \mathrm{~Hz}, 2-\mathrm{H}_{\mathrm{ax}}\right), 5.27$ (dd, $1 \mathrm{H}, J 11.8,5.7 \mathrm{~Hz}$, $\left.4-\mathrm{H}_{\mathrm{ax}}\right), 5.72(\mathrm{~s}, 1 \mathrm{H}, 1-\mathrm{NH}), 6.53-6.44\left(\mathrm{~m}, 2 \mathrm{H}, 6-\mathrm{H}_{\mathrm{Ar}}, 8-\mathrm{H}_{\mathrm{Ar}}\right)$, $6.62\left(\mathrm{~d}, 1 \mathrm{H}, J 7.5 \mathrm{~Hz}, 5-\mathrm{H}_{\mathrm{Ar}}\right), 6.91(\mathrm{td}, 1 \mathrm{H}, J 7.5,0.9 \mathrm{~Hz}$, $\left.7-\mathrm{H}_{\mathrm{Ar}}\right) ;{ }^{13} \mathrm{C}$ NMR $\left(100 \mathrm{MHz}, \mathrm{DMSO}-d_{6}\right) \delta 17.8,21.8,30.8$, 33.7, 41.6, 46.1, 47.4, 113.9, 115.8, 117.8, 125.8, 127.6, 146.4, 174.6; GC-MS (70 eV), $t_{\mathrm{R}} 21.1 \mathrm{~min}, \mathrm{~m} / z$ (relative intensity) $[\mathrm{M}]^{+} 230$ (8), 201 (4), 144 (52), 130 (100), 77 (10). Anal. calcd. for $\mathrm{C}_{14} \mathrm{H}_{18} \mathrm{~N}_{2} \mathrm{O}: \mathrm{C}, 73.01 ; \mathrm{H}, 7.88 ; \mathrm{N}$, 12.16. Found: C, 73.23; H, 7.97; N, 12.09.

cis-1-(6-Methoxy-2-methyl-1,2,3,4-tetrahydroquino-lin-4-yl) pyrrolidin-2-one (3i)

Brown oil; 72\% yield; IR (KBr) v / cm-1 3332, 2962, 1666, 1496, 1434, 1280, 1033, 817; ${ }^{1} \mathrm{H}$ NMR (400 MHz, DMSO- $\left.d_{6}\right) \delta 1.13\left(\mathrm{~d}, 3 \mathrm{H}, J 6.2 \mathrm{~Hz}, 2-\mathrm{CH}_{3}\right), 1.60(\mathrm{q}, 1 \mathrm{H}$, $J 11.9 \mathrm{~Hz}, 3-\mathrm{H}_{\mathrm{ax}}$ ), 1.73 (ddd, 1H, J 11.9, 6.1, $1.80 \mathrm{~Hz}$, 3- $\left.\mathrm{H}_{\mathrm{eq}}\right), 1.96-1.88\left(\mathrm{~m}, 2 \mathrm{H}, 4^{\prime}-\mathrm{H}_{\mathrm{a}}, 4^{\prime}-\mathrm{H}_{\mathrm{b}}\right), 2.43-2.26(\mathrm{~m}, 2 \mathrm{H}$,

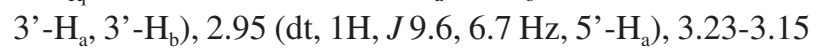
$\left(\mathrm{m}, 1 \mathrm{H}, J\right.$ 9.6, $7.4 \mathrm{~Hz}, 5$ '- $\left.\mathrm{H}_{\mathrm{b}}\right), 3.42-3.34\left(\mathrm{~m}, 1 \mathrm{H}, 2-\mathrm{H}_{\mathrm{ax}}\right)$, 3.59 (s, 3H, 6- $\left.\mathrm{OCH}_{3}\right), 5.27$ (dd, 1H, J 11.9, 6.1 Hz, 4- $\mathrm{H}_{\mathrm{ax}}$ ), 5.31 (s, 1H, 1-NH), 6.22 (d, 1H, J $2.7 \mathrm{~Hz}, 5-\mathrm{H}_{\mathrm{Ar}}$ ), 6.47 (d, $\left.1 \mathrm{H}, J 8.7 \mathrm{~Hz}, 8-\mathrm{H}_{\mathrm{Ar}}\right), 6.59\left(\mathrm{dd}, 1 \mathrm{H}, J 8.7,2.7 \mathrm{~Hz}, 7-\mathrm{H}_{\mathrm{Ar}}\right)$; ${ }^{13} \mathrm{C}$ NMR (100 MHz, DMSO- $\left.d_{6}\right) \delta 17.8,21.9,30.8,33.8$, 41.7, 46.4, 47.7, 55.3, 111.2, 113.9, 115.3, 119.2, 140.7, 150.9, 174.7; GC-MS (70 eV), $t_{\mathrm{R}} 23.2 \mathrm{~min}, \mathrm{~m} / \mathrm{z}$ (relative intensity) $[\mathrm{M}]^{+} 260$ (45), 245 (3), 175 (20), 174 (60), 160 (100), 77 (4). Anal. calcd. for $\mathrm{C}_{15} \mathrm{H}_{20} \mathrm{~N}_{2} \mathrm{O}_{2}$ : C, 69.20; H, 7.74; N, 10.76. Found: C, 69.43; H, 7.58; N, 10.93 .

\section{Results and Discussion}

Optimization process towards the synthesis of 4-acetamidoN-yl-6-acetyl-2-methyl-THQs

The production of 2-methyl-THQs is traditionally performed in organic solvents such as acetonitrile, methanol or aqueous solutions under room temperature or reflux conditions. In our research, we used different $N$-vinyl amides in acid-catalyzed three component $\mathrm{ABB}$ ' reaction under mechano-chemical process to obtain the 2-methylTHQs. Taking into account previous studies ${ }^{15,16}$ and several different reaction parameters used to promote the imino DA reaction, including Lewis and Brønsted acids, we appointed towards the use of Brønsted acids. These ones are inexpensive, easy to handle and generally stable in open-air and water presence.

Keeping in mind these considerations and our two previous studies ${ }^{19,24}$ where we tested some catalyst and 
additives to promove the imino DA, we selected the PMA (heteropolyacid, HPA) as acid catalyst in this new project. In addition, we used the PEG-400 as reaction medium with the PMA. The $p$-aminoacetophenone 1a and $N$-vinyl acetamide 2a were used as our model reaction towards the THQ 3a optimization synthesis.

We took these previous results as the starting point due to the high yields achieved by the use of PMA as catalyst. As you can see, initially the influence over the catalytic activity of PMA or PMA/SiO ${ }_{2}$ in different solvents was evaluated, looking for the best eco-friendly parameters (Table 1). Comparing the PMA conditions with the first results obtained for phthalic acid (PA) in methanol, ${ }^{19}$ we found that the PMA promotes the 3a synthesis in good to excellent yields (60-92\%) (entries 4, 7). Conversely to the entry 3 that any conversion was observed without catalyst, a $5 \mathrm{~mol} \%$ increase of the PMA loading leads to the product yield increase from 60 to $92 \%$ and reduced the reaction time from 4 to $0.5 \mathrm{~h}$ (entry 6). Exploiting the PA results in $\mathrm{MeOH}$ we used the same solvent with PMA observing high yield but still lower than $\mathrm{MeCN}$ assay. Therefore, until now, the PMA showed the best catalytic activity, however, in an attempt to improve the reaction conditions toward a greener process, we prepared the PMA supported on $\mathrm{SiO}_{2}$ $\left(\mathrm{PMA} / \mathrm{SiO}_{2}, 10 \% \mathrm{~m} / \mathrm{m}\right)$ and used as additive. Despite the recycle possibility of this solid acid, through of a simple filtration and washing process, after the second run, it afforded lowers yields. Looking forward to eco-friendly reaction conditions implementation, we also proposed an innocuous reaction media like polyethylene glycol 400 (PEG-400) for the replacement of $\mathrm{MeCN}$, a toxic and expensive solvent. However, the PEG-400 medium did not afford favorable results at two different temperature regimes (entries 10,11). It could be due to the catalyst dispersion in the viscous media and low PMA solubility.

\section{Mechanical energy optimization process}

Continuing with the better reaction conditions search, we wanted to explore other reaction parameters for green imino DA process. In this opportunity, for the first time, the mechanical energy was used to promote the Povarov reaction. In order to carry out the milling imino DA process, we used a high speed vibratory ball mill (HSVBM), a greener chemical method, which is increasingly being implemented in numerous organic reactions. In our research, two different ball and vessel material, agate and steel, were employed. The mechano-chemical energy in the ball mill depends on the apparatus (frequency, movement type) and balls (material, quantity of balls, size and weight). For this HSVBM, only one ball $(\mathrm{d}=51.6 \mathrm{~mm}, 186.25 \mathrm{~g})$ and direct shock interaction in agate dish were used. With

Table 1. Previous and new optimization parameters towards the 4-acetamido- $N$-yl-6-acetyl-2-methyl-THQ 3a synthesis

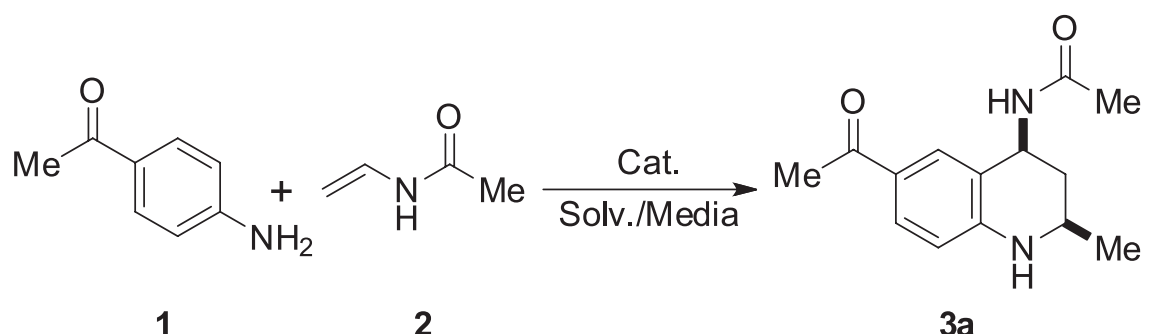

\begin{tabular}{|c|c|c|c|c|c|}
\hline entry & Solvent & Catalyst $^{\mathrm{a}} / \mathrm{mol} \%$ & Temperature $/{ }^{\circ} \mathrm{C}$ & time / h & Yield $^{\mathrm{c}} / \%$ \\
\hline $1^{19}$ & $\mathrm{MeOH}$ & $\mathrm{PA}(50)$ & 50 & 8 & 52 \\
\hline $2^{19}$ & $\mathrm{MeOH}$ & PA (100) & 50 & 8 & 62 \\
\hline 3 & $\mathrm{MeCN}$ & - & $\mathrm{rt}$ & 3 & nil \\
\hline $4^{24}$ & $\mathrm{MeCN}$ & PMA (5) & $\mathrm{rt}$ & 4 & 60 \\
\hline 5 & $\mathrm{MeCN}$ & PMA (5) & 50 & 4 & 74 \\
\hline $6^{24}$ & $\mathrm{MeCN}$ & PMA (10) & $\mathrm{rt}$ & 0.5 & 92 \\
\hline 7 & $\mathrm{MeOH}$ & PMA (10) & $\mathrm{rt}$ & 1 & 85 \\
\hline $8^{24}$ & $\mathrm{MeCN}$ & $\mathrm{PMA} / \mathrm{SiO}_{2}{ }^{\mathrm{b}}$ & 50 & 2 & 50 \\
\hline $9^{24}$ & $\mathrm{MeCN}$ & $\mathrm{PMA} / \mathrm{SiO}_{2}(\text { recycled })^{\mathrm{b}}$ & 50 & 2 & 30 \\
\hline 10 & PEG-400 & PMA (5) & $\mathrm{rt}$ & 2 & nil \\
\hline 11 & PEG-400 & PMA (5) & 60 & 3 & 35 \\
\hline
\end{tabular}

${ }^{\mathrm{a}} \mathrm{mol} \%$ per mol of substrate; ${ }^{\mathrm{c}}$ catalyst $\left(\mathrm{PMA} / \mathrm{SiO}_{2}\right)$ loading $50 \% \mathrm{~m} / \mathrm{m}$ based on aniline's (1) weight (wt.\%); ${ }^{\mathrm{c}} \mathrm{GC}-\mathrm{MS}$ monitored. rt: room temperature. 
Table 2. Reaction time, grinding material, solvent addition and supported catalyst effects on the yield of 3a under HSVBM

\begin{tabular}{|c|c|c|c|c|c|}
\hline entry & Material & Catalyst / $(\mathrm{mol} \%)^{\mathrm{a}}$ & Solvent & time / h & Yield $/ \%$ \\
\hline 1 & agate & 10 & - & 0.25 & 18 \\
\hline 2 & agate & 10 & - & 0.5 & 25 \\
\hline 3 & agate & 10 & - & 0.75 & 27 \\
\hline 4 & agate & 10 & - & 1 & 32 \\
\hline 5 & agate & 10 & - & 2 & 55 \\
\hline 6 & agate & 10 & - & 3 & 65 \\
\hline 7 & agate & 10 & - & 4 & 65 \\
\hline 8 & steel & 10 & - & 3 & 40 \\
\hline 9 & agate & 10 & $\mathrm{H}_{2} \mathrm{O}, 10 \% \mathrm{~m} / \mathrm{m}^{\mathrm{c}}$ & 3 & 10 \\
\hline 10 & agate & 10 & PEG-600, $10 \% \mathrm{~m} / \mathrm{m}^{\mathrm{c}}$ & 3 & 45 \\
\hline 11 & agate & 10 & $\mathrm{SDS}, 10 \% \mathrm{~m} / \mathrm{m}^{\mathrm{c}, \mathrm{d}}$ & 3 & 53 \\
\hline 12 & agate & $\mathrm{PMA} / \mathrm{SiO}_{2}, 10 \% \mathrm{~m} / \mathrm{m}^{\mathrm{c}}$ & - & 3 & 55 \\
\hline
\end{tabular}

${ }^{\mathrm{a}} \mathrm{mol} \%$ per mol of substrate; ${ }^{\mathrm{b}} \mathrm{GC}-\mathrm{MS}$ monitored; 'catalyst (PMA/SiO$)$ loading $50 \% \mathrm{~m} / \mathrm{m}$ based on aniline's (1) weight (mass fraction wt.\%); ${ }^{\mathrm{d} a q u e o u s}$ solution $12 \mathrm{mmol} \mathrm{L}^{-1}, \mathrm{pH}=1$.

the promising results using PMA (Table 1, entry 4) and our interest in the development of solvent-free ABB' imino DA reaction, we focused our attention in a systematic study conducted to optimize the ball milling process (Table 2).

Table 2 shows the results obtained evaluating the influence of the grinding material, reaction time, small quantity of solvent addition (liquid assisted grinding, LAG) and use of supported PMA under HSVBM conditions. The reactions were run at room temperature to the maximum frequency, $60 \mathrm{~Hz}$, evaluating the time of the grinding process through seven different experiments. A promising result was obtained after $60 \mathrm{~min}$, achieving a $32 \%$ conversion to the desired product $3 \mathbf{a}$ based on the activation method tested (Table 2, entry 4). Further, $3 \mathrm{~h}$ were necessary to attain $65 \%$ conversion using an agate ball as grinding material (entry 6). According to our results we could observe that longer reaction times (major than $3 \mathrm{~h}$ ) didn't increase the reaction yield. Taking into account the remarkable obtained results when different kinds of materials (such as stainless steel, zirconium oxide, tempered steel or tungsten carbide) have been used on mechano-chemical process ${ }^{26}$ stainless steel was evaluated as grinding material, showing that this factor has an influence on the domino ABB' decreasing the $3 \mathbf{a}$ product yield. The best result was obtained with the use of agate material as grinding material, while stainless steel is not recommended for the PMA-catalyzed Povarov reaction (entry 8).

On the other hand, the LAG-mechano-chemistry is a well-established branch of solid state mechanical activated reactions, which posits the possible benefit of including a slight amount of solvent that can promote a dramatic increase in the rate of formation product in the reaction media. ${ }^{27}$ For this purpose, $\mathrm{H}_{2} \mathrm{O}$, PEG-600 and SDS were selected and evaluated as solvents in the mechano-chemical treatment (entries 9-11). $\mathrm{H}_{2} \mathrm{O}$ and PEG-600 are recognized as environmental friendly solvents, while SDS $\left(12 \mathrm{mmol} \mathrm{L}^{-1}\right.$, $\mathrm{pH}=1)$ aqueous solution showed, in previous studies, ${ }^{24}$ its remarkable activity in the synthesis of 2-methyl-THQs via ABB' type reactions. Despite benefits, such as reactant(s) dissolution, rapid compound(s) diffusion and subsequent product(s) precipitation, the addition of these three solvents did not produce positive effect, showing lower yields; however, the work-up was easier with the use of SDS solution, due to the rapid precipitation of the $3 \mathbf{a}$ product. In an attempt to reduce the PMA loading, the $\mathrm{PMA} / \mathrm{SiO}_{2}(10 \%$ $\mathrm{m} / \mathrm{m}$ ) acid solid was evaluated. Although the practical workup (dissolution and filtration) became simpler, the conversion was lower than unsupported catalyst (entry 12).

The mechanical treatment (continuous, short act or pulses) has a significant impact on the total mechanical energy input into the reaction mass and therefore, in chemical changes. ${ }^{17}$ In order to provide an experimental support to the mechano-chemical process, we carried out our model reaction on another different type of mill, a high speed mixer ball mill (HSMBM). The mixing mechanical shaking at maximum power $(80 \mathrm{~Hz})$ resulted in a very good conversion of the starting materials into $\mathbf{3 a}$ (Table 3, entry 1). The substrate scope of aryl amines and enamides were also evaluated, showing good results (55-89\% yields, entries 2-7) comparatively with the vibratory milling process. Despite that, there are several drawbacks in the work up procedure, due to the stickiness of the reaction mixture. The high energy input in the milling process obstructs the effective mill because the substrates stick to the walls of the milling jars and milling balls. To avoid this situation, some literature reports ${ }^{28}$ suggest the use of drying and inert materials, such as $\mathrm{MgSO}_{4}$ and $\mathrm{NaCl}$, respectively. Therefore, we used the inert $\mathrm{NaCl}$ in the reaction mixture 
Table 3. Reaction scope with different aryl amines and enamides in the HSMBM process

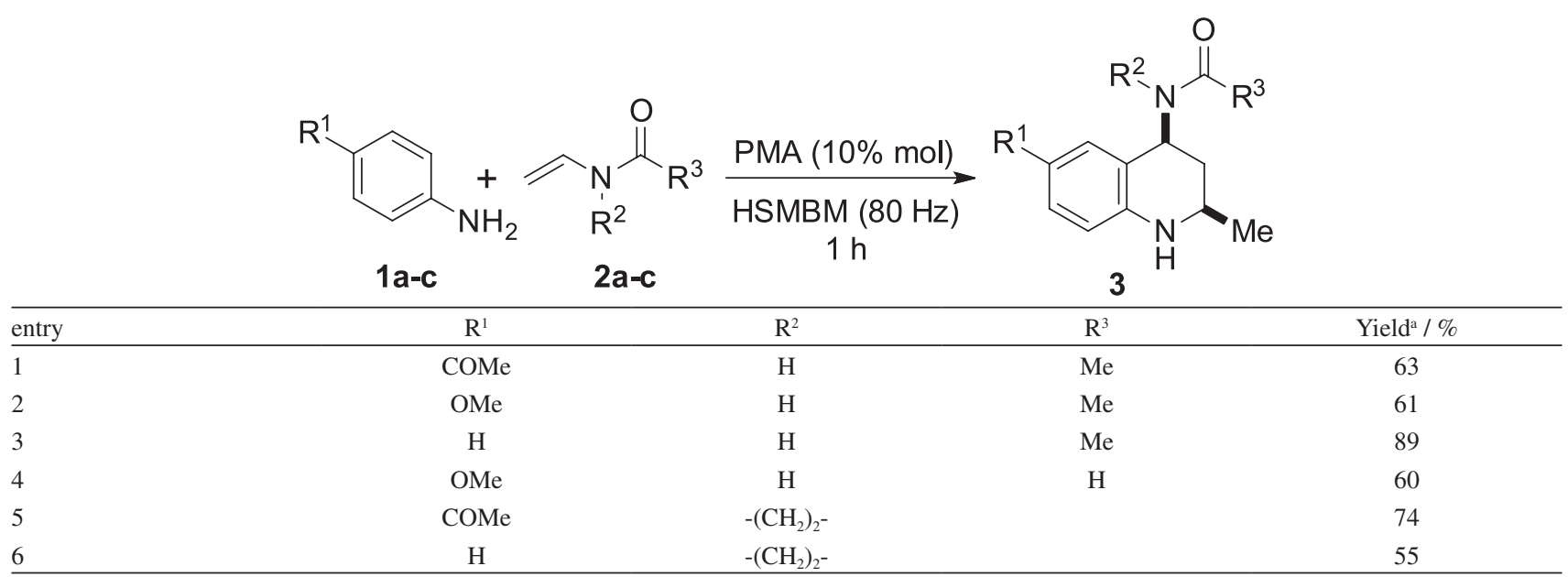

${ }^{\mathrm{a}} \mathrm{GC}-\mathrm{MS}$ monitored.

to make it adequately powdery, but a satisfactory result was not achieved.

\section{Synthesis of 4-acetamido- $N$-yl-6-acetyl-2-methyl-THQ (3a-i)}

Finally, after the optimization process, we choose the HSVBM as energy input system for the PMA-catalyzed mechano-synthesis, especially because its work up is very easy. Thus, we selected several anilines and enamides to evaluate the reaction scope. The final products, 4-amido- $N$ yl-2-methyl-6-substituted THQs 3a-i were easily obtained in good to excellent yields through our greener protocol (Table 4).
The influence of $N$-vinyl amide employed was not relevant according to the yield values, similar with the same aryl amines and different $N$-vinyl amides. On the other hand, the oxygenated functional group on the aniline showed better results than aniline without functionalization. The stereochemistry of all 2-methylTHQs compounds was confirmed by $1 \mathrm{D}$ and $2 \mathrm{D}$ nuclear magnetic resonance (NMR) experiments. The ${ }^{1} \mathrm{H}$ NMR analysis of the THQ products indicated that the structure of the purified diastereoisomers $\mathbf{3 a - i}$ have cis configuration, having the 2-Me/4-N $\left(\mathrm{R}_{2}\right) \mathrm{COR}_{3}$ functional groups as reference. In the same way coupling constants values showed trans disposal between $\mathrm{H}_{3 \mathrm{a}} / \mathrm{H}_{4 \mathrm{a}}\left(J_{H 3 a, H 4 a}>10 \mathrm{~Hz}\right.$, see

Table 4. Optimum yields in the vibratory ball-milling ABB' type imino DA reaction of aryl amines and enamides

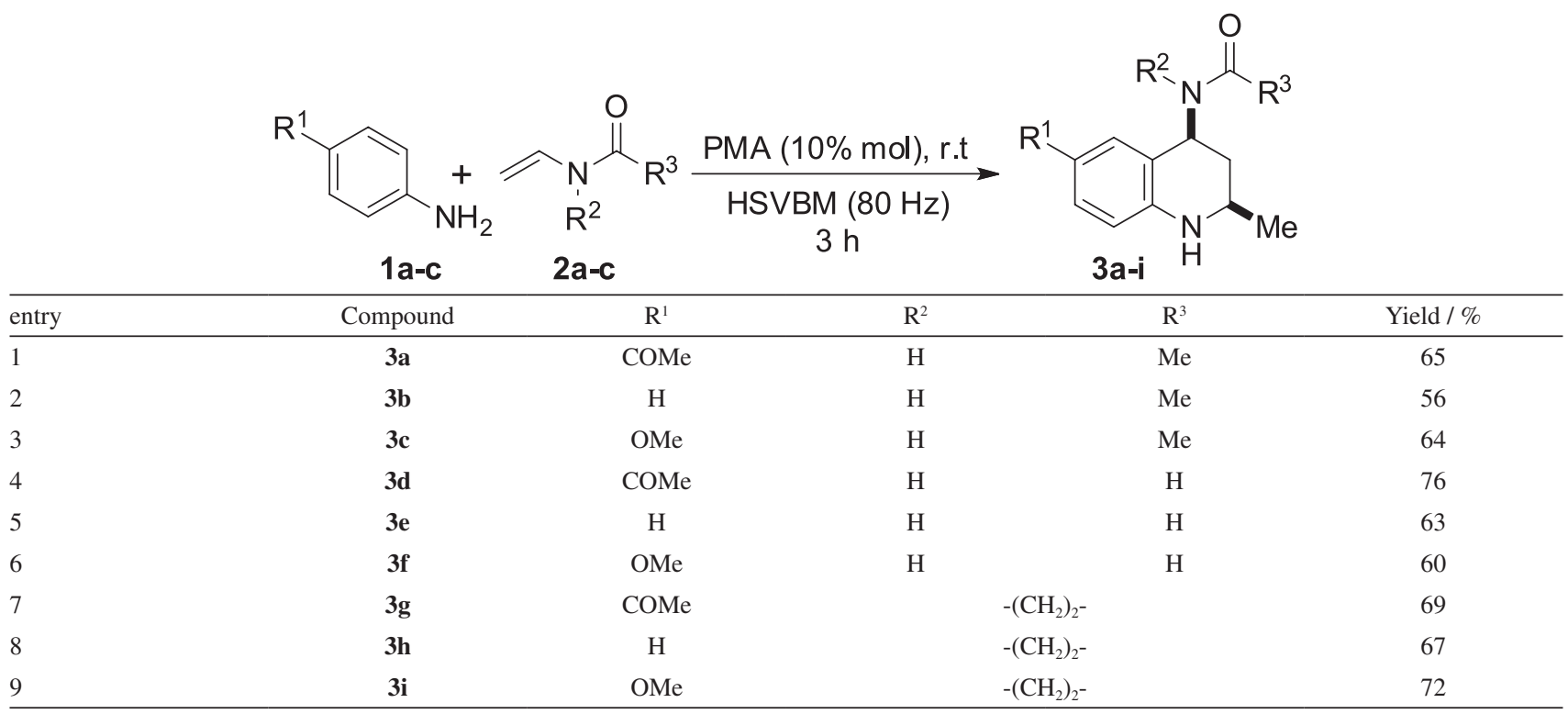


Supplementary Information) and the C-4/C-2 substituents were oriented in equatorial direction. This asseveration was confirmed by single crystal X-ray of the $\mathbf{3 g}$ molecule.

\section{Crystallographic analysis of the $\mathbf{3 g}$ molecule}

A single crystal of $\mathbf{3 g}$ was obtained by carefully vapour diffusion and slow evaporation of its solution in ethanol/ cyclohexane/diethyl ether mixture. The asymmetric unit of the tested compound comprises a molecule of 1-(6-acetyl2-methyl-1,2,3,4-tetrahydroquinolin-4-yl)pyrrolidin-2-one $3 g\left(\mathrm{C}_{16} \mathrm{H}_{20} \mathrm{~N}_{2} \mathrm{O}_{2}\right)$, which crystallizes in the orthorhombic centrosymmetric system with space group Pbca (No. 61).

The molecular structure of the compound is shown in Figure 1. This molecule contains a six-membered ring (N7-C6-C5-C4-C9-C8) fused to the aromatic system which have an envelope conformation with atom $\mathrm{C} 9$ as the flap with puckering parameters $\mathrm{Q}=0.4905(17) \AA$, $\theta=50.97(19)^{\circ}$ and $\varphi=249.4(2)^{\circ}$. The five-membered ring (N3-C2-C20-C19-C18) adopts a twisted conformation with puckering parameters $\mathrm{Q}(2)=0.2441(18) \AA$ and $\varphi=117.7(4)^{\circ}$.

In the crystal structure, the molecules are linked by intermolecular interactions of different strength. In the

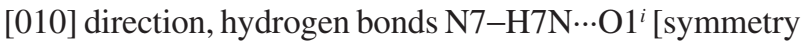
code: $(i)-\mathrm{x}+3 / 2, \mathrm{y}+1 / 2$ ] join molecules into chains that are further connected by weaker $\mathrm{C} 17-\mathrm{H} 17 \mathrm{C} \cdots \mathrm{O} 16^{i i}$ [symmetry code: (ii) $\mathrm{x}-1 / 2, \mathrm{y},-\mathrm{z}+3 / 2$ ] hydrogen bonds along [001] direction, forming (001) sheets (Figure 2). These sheets are connected in the [100] and [001] directions by a mixture of dipolar and van der Waals forces (for an extensive single crystal analysis, see Supplementary Information).

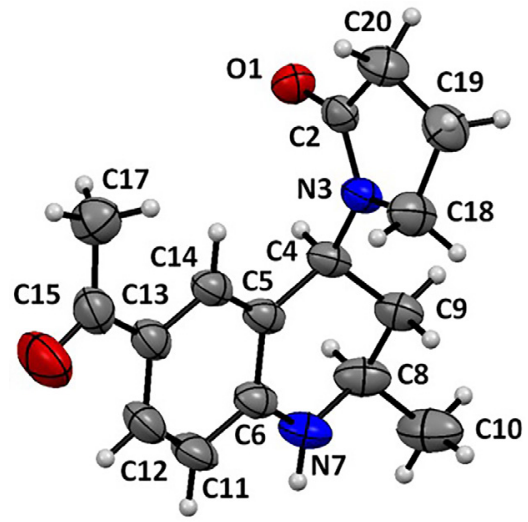

Figure 1. The $\mathbf{3 g}$ structure showing the anisotropic displacement ellipsoids drawn at the $50 \%$ probability level (hydrogen atoms are depicted as spheres with arbitrary radii).

The PMA $\left(\mathrm{H}_{3} \mathrm{PMo}_{12} \mathrm{O}_{40}\right)$ is one typical example of Keggin-type HPA and is widely used as homogeneous and heterogeneous oxidant and acid catalysts performing efficiently industrial processes and transformations in organic chemistry. ${ }^{27}$ However, its behavior as oxidant agents or solid acid catalysts has not been clearly defined. Some general topics should be discussed here, taking into account that the HPAs are strong Brønsted acids (superacids), wellknown proton conductors and its nature acidity is purely Brønsted defined.

In spite of we don't have any experimental evidence about the PMA acidity, previous studies discuss this topic of various HPAs, including its acidity properties in solution and solid state, statement for this compounds fully dissociation in aqueous solution and extremely proton mobility in solid state (this property is highly related with its catalytic activity). ${ }^{29}$ Comparing both HPAs used in ABB' DA reaction, PMA (our research $)$ and PWA,${ }^{18}$ the PMA $\left(p K_{1}=2.0\right)$ is less acid than
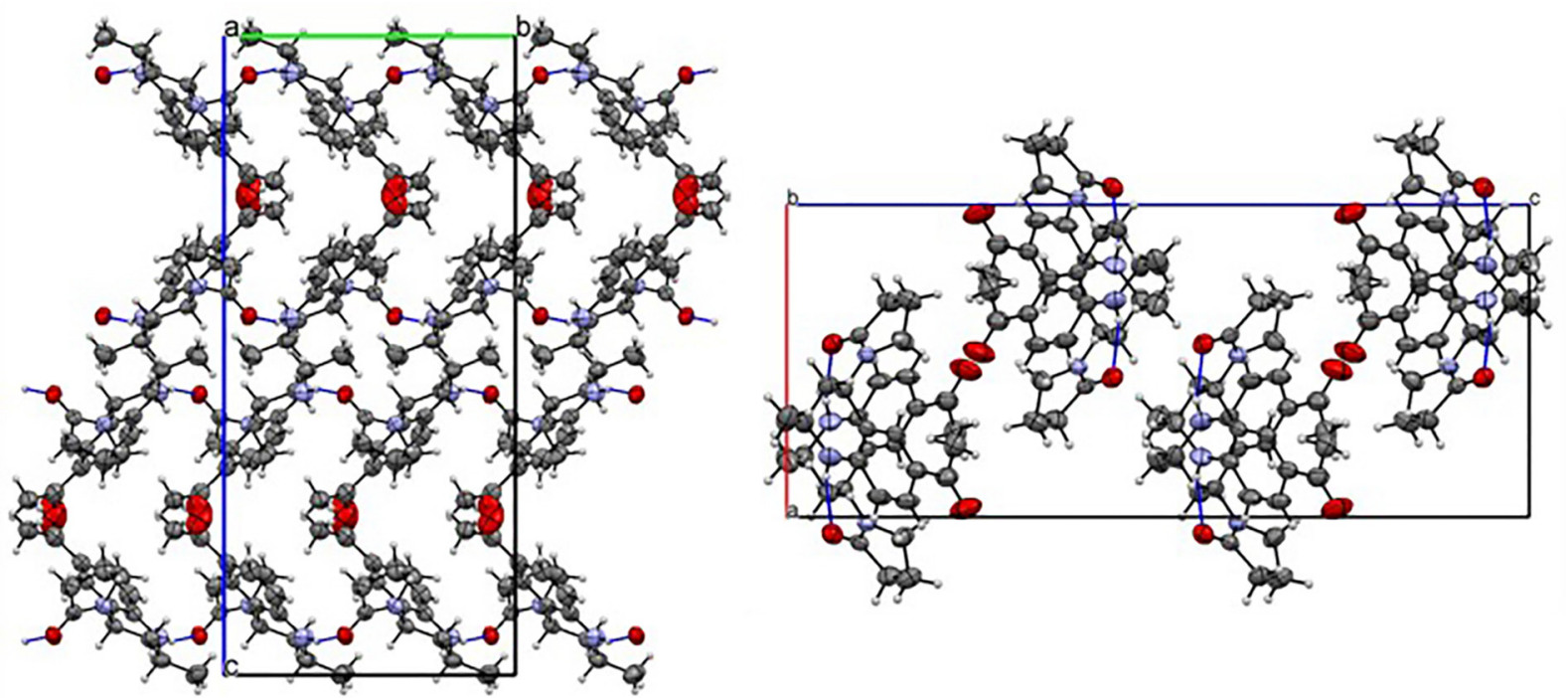

Figure 2. Packing of the $\mathrm{C}_{16} \mathrm{H}_{20} \mathrm{~N}_{2} \mathrm{O}_{2}$ compound viewed along [100] and [010] directions, showing hydrogen bonded chains of molecules. 
PWA $\left(p \mathrm{~K}_{1}=1.6\right)$, but both are stronger than mineral acids $\left(\mathrm{H}_{2} \mathrm{SO}_{4},\left(p \mathrm{~K}_{1}=6.6\right) ; \mathrm{HCl},\left(p \mathrm{~K}_{1}=4.3\right) ; \mathrm{HNO}_{3},\left(p \mathrm{~K}_{1}=9.4\right)\right) .{ }^{30}$ On the other hand, the HPAs also exhibit fast reversible multielectron redox behavior under mild conditions. ${ }^{31}$ In the reaction with solvent $(\mathrm{MeCN})$, both catalysts (PMA and PWA) work well ${ }^{18}$ as it was expected, however, few evidences about the catalityc activity in solvent free Diels-Alder reaction have been reported. In this way, having into account the importance of proton mobility and acidity of PMA in the catalytic process, we performed the model reaction using another potentially molybdenum-based catalyst, APM (ammonium phosphomolybdate, $\left(\mathrm{NH}_{4}\right)_{3} \mathrm{PMo}_{12} \mathrm{O}_{40}$, Scheme 1). ${ }^{32}$ The APM conserves the heteropoly-anion (core of the Keggin structure) and the counter-ion has been replaced by $\mathrm{NH}_{4}^{+}$ions. Interestingly, under the same freesolvents conditions (model reaction, catalyst $10 \mathrm{~mol} \%$, agate material, free-solvent, $80 \mathrm{~Hz}, \mathrm{HSVBM}$ ) the APM did not promote the model reaction.

In this case, the substitution of this cation causes the loss of the proton conductivity efficiency and therefore, didn't show any catalytic properties. Moreover, it could help us to understand that both, PMA Keggin structure and Brønsted acid nature, are necessary for the decisive role in this catalytic process. In the same way, the noteworthy PMA oxidant activity was visually checked by the "heteropoly blue anion" formation during PMA-catalyzed model reaction performance (see Supplementary Information). It proves that the one or two electron transfer reversible process (through an electron transfer reaction, ETR) can occur $^{33}$ during the reaction and could mean that the reaction mechanism is more complex than some authors have proposed with similar HPA catalyst, like PWA. ${ }^{18}$ In that case, the authors claimed that the PWA central atom, W (Tungsten), has higher oxidation state, strong electrophilic character and Lewis acidity. However, they didn't mention anything about the Keggin-type structure and Brønsted acid properties of PWA, even taking into account that the PWA is more acid than the PMA, and that the PMA reduction potential is lower than the PWA. ${ }^{14}$

Thus, the powerful catalytic HPA action demonstrated in this paper seems to be related with suitable acidity and redox potential in both sequential proposed steps, "Mannich-like" reaction and imino DA cycloaddition process. It could occur via an intramolecular electrophilic substitution of a carbenium ion or a cation-radical species (stepwise mechanisms) (a) and (b) or a concerted mechanism (c) via an asynchronous transition state ${ }^{11}$ (Scheme 1). Some experimental works to clarify the complex domino imino DA mechanism are underway in our laboratory, and the results will be published posteriorly.

\section{Conclusion}

In summary, we developed a new methodology to induce the acid Brønsted or oxidant catalysed imino DA reaction under two mechano-chemical processes

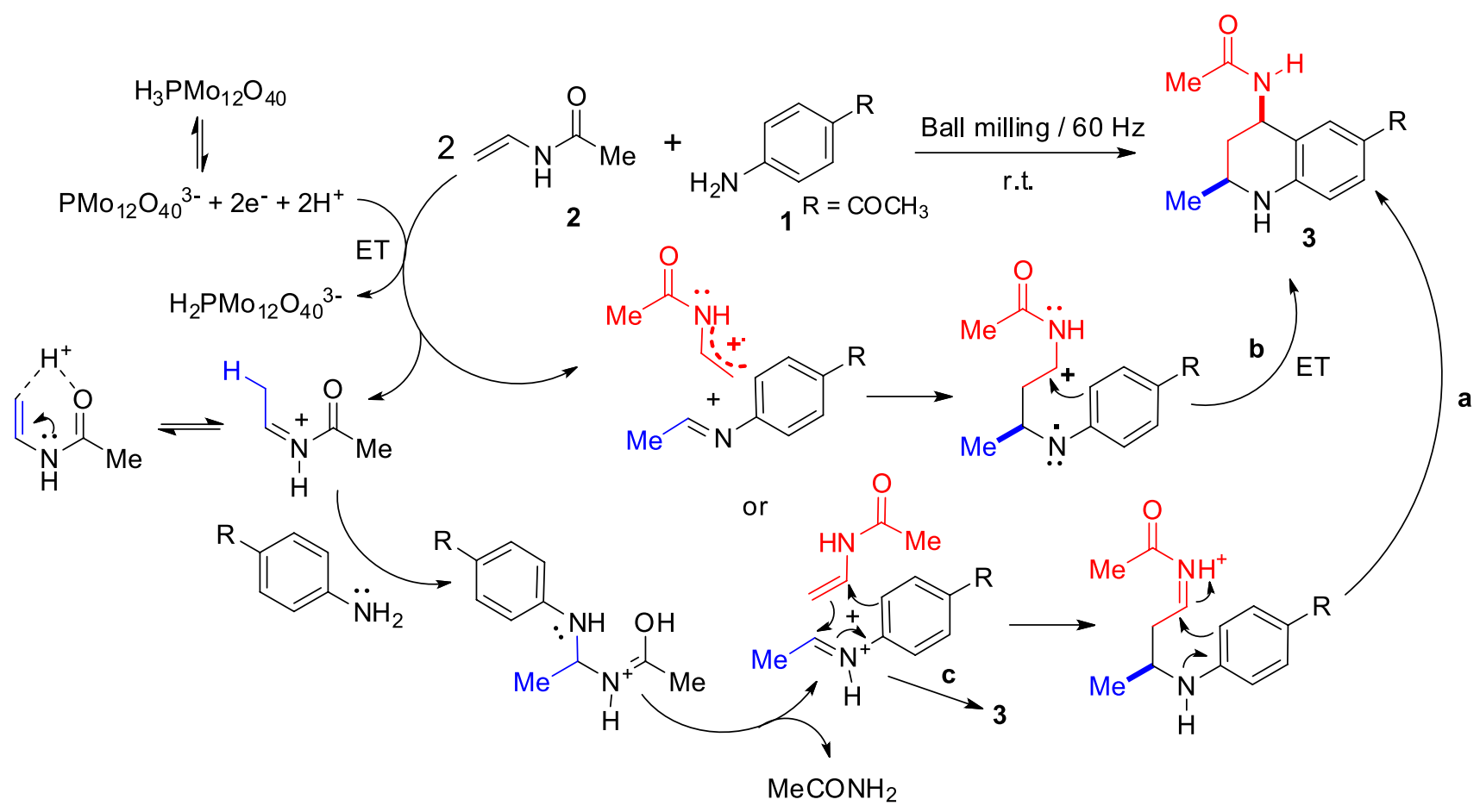

Scheme 1. Proposed mechanism to the PMA catalysed ABB' Povarov reaction. 
as energy input. In our case, the HSVBM was the best system to promote the formation of diverse 2-Me-1,2,3,4tetrahydroquinolines $\mathbf{3 a - i}$ in diastereoselective manner. Additionally, we checked the importance of the counter ion $\mathrm{H}^{+}$presence in the catalyst, changing this $\mathrm{H}^{+}$to $\mathrm{NH}_{4}{ }^{+}$ion in the PMA, where we did not observe any conversion in the APM-catalyzed reaction. It could be due to the proton conductor efficiency lack.

\section{Supplementary Information}

Supplementary data (experimental details and analytical data for all compounds as well as the ${ }^{1} \mathrm{H},{ }^{13} \mathrm{C}$ and $2 \mathrm{D}$ NMR

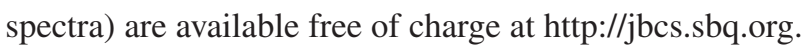
br as PDF file.

\section{Acknowledgments}

Financial support from Patrimonio Autónomo Fondo Nacional de Financiamiento para la Ciencia, la Tecnología y la Innovación, Francisco José de Caldas, contract RC0346-2013, is gratefully acknowledged. D. R. M. A. thanks COLCIENCIAS for the fellowship. C. A. M. B thanks VIE of Universidad industrial de Santander (Colombia) for the financial support.

\section{References}

1. Wang, G. W.; Chem. Soc. Rev. 2013, 42, 7668; Boldyreva, E.; Chem. Soc. Rev. 2013, 42, 7719; Zhang, Z.; Peng, Z.; Hao, M.; Gao, J.; Synlett 2010, 19, 2895.

2. Hsu, C.; Lai, C.; Chiu, S.; Tetrahedron 2009, 65, 2824.

3. McKissic, K. S.; Caruso, J. T.; Blair, R. G.; Mack, J.; Green Chem. 2014, 16, 1628.

4. Sridharan, V.; Avendaño, C.; Menéndez, J. C.; Tetrahedron 2007, 63, 673; Tan, Y.-J.; Zhang, Z.; Wang, F.-J.; Wu, H.-H.; Li, Q.-H.; RSC Adv. 2014, 4, 35635.

5. Anastas, P. T.; Warner J. C.; Green Chemistry: Theory and Practice; Oxford University Press: Oxford, 2000.

6. Nicolaou, K.C.; Snyder, S.A.; Montagnon, T.; Vassilikogiannakis, G.; Angew. Chem. Int. Ed. 2002, 41, 1668.

7. Boger, D.; Weinreb, S.; Organic Chemistry, Hetero Diels-Alder Methodology in Organic Synthesis; Academic Press, Inc.: San Diego, 1987.

8. Buonora, P.; Olsen, J.-C.; Oh, T.; Tetrahedron 2001, 57, 6099.

9. Povarov, L. S.; Russ. Chem. Rev. 1967, 36, 656-670.

10. Sridharan, V.; Suryavanshi, P. A.; Menéndez, J. C.; Chem. Rev. 2011, 111, 7157.

11. Kouznetsov, V. V.; Tetrahedron 2009, 65, 2721.

12. Cope, H.; Mutter, R.; Heal, W.; Pascoe, C.; Brown, P.; Pratt, S.; Chen, B.; Eur. J. Med. Chem. 2006, 41, 1124.
13. Leeson, P. D.; Carling, R. W.; Moore, K. W.; Moseley, A. M.; Smith, J. D.; Stevenson, G.; Chan, R.; Foster, A. C.; Grimwood, S.; Kemp, J. A.; Marshall, A. G.; Hoogsteen, K.; J. Med. Chem. 1992, 35, 1954.

14. Liu, J.; Wang, Y.; Sun, Y.; Marshall, D.; Miao, S.; Tonn, G.; Anders, P.; Tocker, J.; Tang, H. L.; Medina, J.; Bioorg. Med. Chem. Lett. 2009, 19, 6840.

15. Srinivasa, A.; Mahadevan, K. M.; Hulikal, V.; Synth. Commun. 2008, 39, 93.

16. Kavitha, C. S.; Hosamani, K. M.; Harisha, R. S.; Can. J. Chem. 2010, 88, 443.

17. Varma, R. S.; Green Chem. 2008, 10, 1129; Sartori, G.; Bigi, F.; Maggi, R.; Mazzacani, A.; Oppici, G.; Eur. J. Org. Chem. 2001, 13, 2513.

18. Kavitha, C. S.; Hosamania, K. M.; Seetharamareddy, H. R.; Synth. Commun. 2011, 41, 33.

19. Kouznetsov, V. V.; Merchan Arenas, D. R.; OrtizAreniz, C.; Meléndez Gómez, C.; Synthesis 2011, 24, 4011; Merchan Arenas, D. R.; Rojas Ruíz, F. A.; Kouznetsov, V. V.; Tetrahedron Lett. 2011, 52, 1388; Kouznetsov, V. V; Gómez Melendez, C. M.; Parada Luna, L. K.; Bermudez, J. H.; Méndez Vargas, L. Y.; Acevedo Muñoz, A.; Mol. Divers. 2011, 15, 1007.

20. Han, B.; Jia, X.-D.; Jin, X.-L.; Zhou, Y.-L.; Yang, L.; Liu, Z.-L.; Yu, W.; Tetrahedron Lett. 2006, 47, 3545.

21. Savitha, G.; Perumal, P. T.; Tetrahedron Lett. 2006, 47, 3589.

22. Chandrashekarappa, K. K. H.; Mahadevan, K. M.; Manjappa, K. B.; Tetrahedron Lett. 2013, 54, 1368.

23. Boddula, R.; Srinivasan, P.; Catal. Commun. 2013, 30, 56.

24. Merchán Arenas, D. R.; Martínez Bonilla, C. A.; Kouznetsov, V. V.; Org. Biomol. Chem. 2013, 11, 3655.

25. Kishore Kumar, G. D.; Baskaran, S.; J. Org. Chem. 2005, 70, 4520 .

26. Schneider, F.; Stolle, A.; Ondruschka, B.; Hopf, H.; Org. Process Res. Dev. 2009, 13, 44.

27. Bowmaker, G. A.; Chem. Commun. 2013, 49, 334; Bowmaker, G. A.; Hanna, J. V.; Skelton, B. W.; Chem. Commun. 2009, 2168.

28. Kozhevnikov, I. V.; Chem. Rev. 1998, 98, 171; Firouzabadi, H.; Jafari, A. A.; J. Iran. Chem. Soc. 2005, 2, 85.

29. Bardin, B. B.; Bordawekar, S. V.; Neurock, M.; Davis, R. J.; J. Phys. Chem. B 1998, 102, 10817.

30. Marchal-Roch, C.; Millet, J. M.; Comptes Rendus l'Académie des Sci. - Ser. IIC - Chem. 2001, 4, 321.

31. Sadakane, M.; Steckhan, E.; Chem. Rev. 1998, 98, 219.

32. For APM preparation, see: Dermeche, L.; Thouvenot, R.; Hocine, S.; Rabia, C.; Inorganica Chim. Acta 2009, 362, 3896; Cavani, F.; Mezzogori, R.; Pigamo, A.; Trifirò, F.; Comptes Rendus l'Académie des Sci. - Ser. IIC - Chem. 2000, 3, 523.

33. Kozhevnikov, I. V.; Russ. Chem. Rev. 1987, 56, 811.

Submitted: February 4, 2016 Published online: April 15, 2016 